\title{
On two fractional differential inclusions
}

\author{
Dumitru Baleanu ${ }^{1,2^{*}} \mathbb{D}$, Vahid Hedayati ${ }^{3}$, Shahram Rezapour ${ }^{3}$ and Maysaa' Mohamed Al Qurashi ${ }^{4}$
}

${ }^{*}$ Correspondence: dumitru@
cankaya.edu.tr
${ }^{1}$ Department
of Mathematics, Cankaya
University, Ogretmenler Cad.
14,06530 Balgat, Ankara,
Turkey
Full list of author information
is available at the end of the
article

*Correspondence: dumitru@ University, Ogretmenler Cad. 14, 06530 Balgat, Ankara, article

\begin{abstract}
We investigate in this manuscript the existence of solution for two fractional differential inclusions. At first we discuss the existence of solution of a class of fractional hybrid differential inclusions. To illustrate our results we present an illustrative example. We study the existence and dimension of the solution set for some fractional differential inclusions.

Keywords: Fixed point, Fractional hybrid differential inclusions, Dimension solution set
\end{abstract}

\section{Background}

As you know, fractional dynamical systems be used in modeling of some real processes and there are many published works about the existence of solutions for many fractional differential equations (see for example Baleanu et al. 2013a, b, c; Chai 2013 and the references therein) and inclusions (see for example, Benchohra and Hamidi 2010; Agarwal et al. 2013; Ahmad et al. 2013; Nieto et al. 2013; Ouahab 2008; Phung and Truong 2013; Bragdi et al. 2013 and the references therein). For finding more details about elementary notions and definitions of fractional differential equations and inclusions one can study well-known books (see for example Aubin and Ceuina 1984; Deimling 1992; Kilbas et al. 2006; Kisielewicz 1991; Podlubny 1999). Recently, it has been published many useful works about modeling of fractional differential equations via providing different applications in some fields (see for example Atangana 2016; Atangana and Alkahtani 2016; Atangana and Koca 2016a, b). In this article, we first review the existence solution for the fractional hybrid derivative inclusion ${ }^{c} D^{\alpha}\left(\frac{y(s)}{g\left(s, y(s), I^{\alpha} y(s), \ldots, I^{\left.\alpha_{n} y(s)\right)}\right.}\right) \in G\left(s, y(s), I^{\beta_{1}} y(s), \ldots, I^{\beta_{k}} y(s)\right)$ with boundary conditions $y(0)=y_{0}$ and $y(1)=y_{1}$, where $1<\alpha \leq 2, \alpha_{1}, \ldots, \alpha_{n}>0, \beta_{1}, \ldots, \beta_{k}>0, y_{0}, y_{1} \in \mathbb{R},{ }^{c} D^{\alpha}$ denotes Caputo fractional derivative of order $\alpha, g: J \times \mathbb{R}^{n} \rightarrow \mathbb{R}-\{0\}$ is continuous and $G: J \times \mathbb{R}^{k} \rightarrow \mathcal{P}(\mathbb{R})$ is a multifunction via some properties. Also, we review existence and dimension of the solution set of fractional derivative inclusion

$$
{ }^{c} D^{\alpha} y(s) \in G\left(s, y(s),(\phi y)(s),(\psi y)(s),{ }^{c} D^{\beta_{1}} y(s), \ldots,{ }^{c} D^{\beta_{k}} y(s), I^{\gamma_{1}} y(s), \ldots, I^{\gamma_{k}} y(s)\right)
$$

with boundary condition $y(0)+\sum_{i=1}^{k}{ }^{c} D^{\beta_{i}} y(1)+\sum_{i=1}^{k} I^{\gamma_{i}} y(1)=0$, where $0<\beta_{i}<\alpha \leq 1$ and $0<\gamma_{i}<1$ for $i=1, \ldots, k, G: J \times \mathbb{R}^{2 k+3} \rightarrow \mathcal{P}(\mathbb{R})$ is a multifunction via some properties, $\gamma, \lambda: J \times J \rightarrow[0, \infty)$ are two mappings with the properties

(c) 2016 The Author(s). This article is distributed under the terms of the Creative Commons Attribution 4.0 International License (http://creativecommons.org/licenses/by/4.0/), which permits unrestricted use, distribution, and reproduction in any medium, provided you give appropriate credit to the original author(s) and the source, provide a link to the Creative Commons license, and indicate if changes were made. 
$\sup _{s \in J}\left|\int_{0}^{s} \lambda(s, t) d s\right|<\infty$ and $\sup _{s \in J}\left|\int_{0}^{s} \gamma(s, t) d s\right|<\infty$ and the functions $\phi$ and $\psi$ are defined by $(\phi y)(s)=\int_{0}^{s} \gamma(s, t) y(t) d t$ and $(\psi y)(s)=\int_{0}^{s} \lambda(s, t) y(t) d t$.

\section{Preliminaries}

Suppose that $(\mathcal{X}, d)$ be a metric space. Denote by $\mathcal{P}(\mathcal{X})$ and $2^{\mathcal{X}}$ the class of all subsets and the class of all nonempty subsets of $\mathcal{X}$ respectively. Here, $\mathcal{P}_{c l}(\mathcal{X}), \mathcal{P}_{b d}(\mathcal{X})$, $\mathcal{P}_{c v}(\mathcal{X})$ and $\mathcal{P}_{c p}(\mathcal{X})$ denote the class of all closed, bounded, convex and compact subsets of $\mathcal{X}$ respectively. A mapping $\mathcal{Q}: \mathcal{X} \rightarrow 2^{\mathcal{X}}$ is called a multifunction on $\mathcal{X}$ and $x \in \mathcal{X}$ is called a fixed point of $\mathcal{Q}$ whenever $x \in \mathcal{Q} x$ (Deimling 1992). A multifunction $\mathcal{Q}: \mathcal{X} \rightarrow \mathcal{P}(\mathcal{X})$ is called lower semi-continuous whenever the set $\mathcal{Q}^{-1}(A):=\{x \in \mathcal{X}: \mathcal{Q} x \cap A \neq \emptyset\}$ is open for each open subset $A$ of $\mathcal{X}$ (Kisielewicz 1991). If the set $\{x \in \mathcal{X}: \mathcal{Q} \mathcal{X} \subset A\}$ is open for each open set $A$ of $\mathcal{X}$, then we say that $\mathcal{Q}$ is upper semi-continuous (Kisielewicz 1991). A multifunction $\mathcal{Q}: \mathcal{X} \rightarrow \mathcal{P}(\mathcal{X})$ is called compact whenever $\overline{\mathcal{Q}(S)}$ is a compact for each bounded subsets $S$ of $\mathcal{X}$ (Aubin and Ceuina 1984). A multifunction $\mathcal{Q}: J \rightarrow \mathcal{P}_{c l}(\mathbb{R})$ is said to be measurable whenever the function $s \mapsto d(y, \mathcal{Q}(s))=\inf \{|y-z|: z \in \mathcal{Q}(s)\}$ is measurable for all $y \in \mathbb{R}$ and $s \in J=[0,1]$ (Deimling 1992). The Pompeiu-Hausdorff metric $H=H_{d}$ on $2^{\mathcal{X}} \times 2^{\mathcal{X}}$ into $[0, \infty)$ is defined by $H(\mathcal{A}, \mathcal{B})=\max \left\{\sup _{a \in \mathcal{A}} d(a, \mathcal{B}), \sup _{b \in \mathcal{B}} d(\mathcal{A}, b)\right\}$, where $d(\mathcal{A}, b)=\inf _{a \in \mathcal{A}} d(a ; b)$ (Berinde and Pacurar 2013). Then $\left(\mathcal{P}_{b d, c l}(\mathcal{X}), H\right)$ is a metric space and $\left(\mathcal{P}_{c l}(\mathcal{X}), H\right)$ is a generalized metric space (Berinde and Pacurar 2013). A multifunction $\mathcal{Q}: \mathcal{X} \rightarrow \mathcal{P}_{c l}(\mathcal{X})$ is called a contraction whenever there exists $\gamma \in(0,1)$ such that $H_{d}(\mathcal{Q}(x), \mathcal{Q}(y)) \leq \gamma d(x, y)$ for all $x, y \in \mathcal{X}$ (Covitz and Nadler 1970). Covitz and Nadler (1970) proved that each closed valued contractive multifunction on a complete metric space has a fixed point. We say that $\mathcal{Q}: J \times \mathbb{R}^{k} \rightarrow 2^{\mathbb{R}}$ is a Caratheodory multifunction whenever $s \mapsto \mathcal{Q}\left(s, x_{1}, x_{2}, \ldots, x_{k}\right)$ is measurable for all $x_{1}, x_{2}, \ldots, x_{k} \in \mathbb{R}$ and $\left(x_{1}, x_{2}, \ldots, x_{k}\right) \mapsto \mathcal{Q}\left(s, x_{1}, x_{2}, \ldots, x_{k}\right)$ is an upper semi-continuous map for almost all $s \in J$ (see Aubin and Ceuina 1984; Deimling 1992; Kisielewicz 1991). Also, a Caratheodory multifunction $\mathcal{Q}: J \times \mathbb{R}^{k} \rightarrow 2^{\mathbb{R}}$ is called $L^{1}$-Caratheodory whenever for each $\rho>0$ there exists $\phi_{\rho} \in L^{1}\left(J, \mathbb{R}^{+}\right)$such that

$$
\left\|\mathcal{Q}\left(s, x_{1}, x_{2}, \ldots, x_{k}\right)\right\|=\sup \left\{|v|: v \in \mathcal{Q}\left(s, x_{1}, x_{2}, \ldots, x_{k}\right)\right\} \leq \phi_{\rho}(s)
$$

for all $\left|x_{1}\right|,\left|x_{2}\right|, \ldots,\left|x_{k}\right| \leq \rho$ and for almost all $s \in J$ (see Aubin and Ceuina 1984; Deimling 1992; Kisielewicz 1991).

Lemma 1 (Deimling 1992) If $G: \mathcal{X} \rightarrow \mathcal{P}_{c l}(\mathcal{Y})$ is upper semi-continuous, then $\operatorname{Gr}(G)$ is a closed subset of $\mathcal{X} \times \mathcal{Y}$. If $G$ is completely continuous and has a closed graph, then it is upper semi-continuous.

Lemma 2 (Lasota and Opial 1965) Suppose that $\mathcal{X}$ is a Banach space, $G: J \times \mathcal{X} \rightarrow \mathcal{P}_{c p, c v}(\mathcal{X})$ an $L^{1}$-Caratheodory multivalued and $\Theta$ a linear continuous mapping from $L^{1}(J, \mathcal{X})$ to $C(J, \mathcal{X})$. Then the mapping $\Theta o S_{G}: C(J, \mathcal{X}) \rightarrow \mathcal{P}_{c p, c \nu} C(J, \mathcal{X})$ defined by $\left(\Theta o S_{G}\right)(x)=\Theta\left(S_{G, x}\right)$ is a closed graph mapping in $C(J, \mathcal{X}) \times C(J, \mathcal{X})$.

Theorem 3 (Dhage 2006) Suppose that $\mathcal{X}$ is a Banach algebra space, $S \in \mathcal{P}_{b d, c l, c v}(\mathcal{X})$ and $\mathcal{A}: S \rightarrow \mathcal{P}_{c l, c v, b d}(\mathcal{X})$ and $\mathcal{B}: S \rightarrow \mathcal{P}_{c p, c v}(\mathcal{X})$ two multifunctions satisfying the following conditions 
1. $\mathcal{A}$ is Lipschitz with a Lipschitz constant $k$,

2. $\mathcal{B}$ is upper semi-continuous and compact,

3. $\mathcal{A x B} x$ is a convex subset $S$ for all $x \in S$,

4. $M k<1$, where $M=\|\mathcal{B}(S)\|=\sup \{\|\mathcal{B} x\|: x \in S\}$.

Then, there exists $y \in S$ such that $y \in \mathcal{A} y \mathcal{B} y$.

Lemma 4 (Agarwal et al. 2013) Suppose that $G:[0,1] \rightarrow \mathcal{P}_{c p, c v}(\mathbb{R})$ is a measurable map such that the Lebesgue measure $\mu$ of the set $\{s: \operatorname{dim} G(s)<1\}$ is zero. Then there are arbitrarily many linearly independent measurable selections $x_{1}(),. \ldots, x_{m}($.$) of G$.

Theorem 5 (Agarwal et al. 2013) Suppose that $C$ is a nonempty closed convex subset of Banach space $\mathcal{X}$. Let $G: C \rightarrow \mathcal{P}_{c p, c v}(C) b$ a $\gamma$-contraction. If $\operatorname{dim} G(x) \geq m$ for all $x \in C$, then $\operatorname{dim} \operatorname{Fix}(G) \geq m$.

\section{Main results}

First, we review the fractional hybrid differential inclusion

$$
{ }^{c} D^{\alpha}\left(\frac{y(s)}{g\left(s, y(s), I^{\alpha_{1}} y(s), \ldots, I^{\alpha_{n}} y(s)\right)}\right) \in G\left(s, y(s), I^{\beta_{1}} y(s), \ldots, I^{\beta_{k}} y(s)\right)
$$

with the boundary conditions $y(0)=y_{0}$ and $y(1)=y_{1}$, where $1<\alpha \leq 2, \alpha_{1}, \ldots, \alpha_{n}>0$, $\beta_{1}, \ldots, \beta_{k}>0, \quad y_{0}, y_{1} \in \mathbb{R},{ }^{c} D^{\alpha}$ denotes Caputo fractional derivative of order $\alpha$, $g: J \times \mathbb{R}^{n} \rightarrow \mathbb{R}-\{0\}$ is continuous and $G: J \times \mathbb{R}^{k} \rightarrow \mathcal{P}(\mathbb{R})$ is a multifunction via some properties.

Lemma 6 Suppose that $x \in C(J, \mathbb{R}), \alpha \in(1,2]$ and $\alpha_{1}, \ldots, \alpha_{n}>0$. The unique solution of the fractional differential problem ${ }^{c} D^{\alpha}\left(\frac{y(s)}{g\left(s, y(s), I^{\alpha_{1}} y(s), \ldots, I^{\alpha_{n}} y(s)\right)}\right)=x(s)$ with the boundary value conditions $y(0)=y_{0}$ and $y(1)=y_{1}$ is given by

$$
\begin{aligned}
y(s) & =g\left(s, y(s), I^{\alpha_{1}} y(s), \ldots, I^{\alpha_{n}} y(s)\right) \\
& \times\left[I^{\alpha} x(s)+\frac{(1-s) y_{0}}{g(0, y(0), \underbrace{0, \ldots, 0}_{n})}+\frac{s y_{1}}{f\left(1, y(1), I^{\alpha_{1}} y(1), \ldots, I^{\alpha_{n}} y(1)\right)}-s I^{\alpha} x(1)\right] .
\end{aligned}
$$

Proof The general solution of the equation ${ }^{c} D^{\alpha}\left(\frac{y(s)}{g\left(s, y(s), I^{\alpha} 1 y(s), \ldots, I^{\alpha_{n}} y(s)\right)}\right)=x(s)$ is $y(s)=g\left(s, y(s), I^{\alpha_{1}} y(s), \ldots, I^{\alpha_{n}} y(s)\right)\left[I^{\alpha} x(s)+c_{0}+c_{1} s\right]$, where $c_{0}, c_{1} \in \mathbb{R}$ are arbitrary constants (see Kilbas et al. 2006; Podlubny 1999). By using the boundary conditions,

we $\quad$ get $\quad y(0)=g(0, y(0), \underbrace{0, \ldots, 0}_{n}) c_{0}=y_{0} \quad$ and $\quad y(1)=g\left(1, y(1), I^{\alpha_{1}} y(1), \ldots, I^{\alpha_{n}} y(1)\right)$ $\left(I^{\alpha} x(1)+c_{0}+c_{1}\right)=y_{1}$. Hence, $\quad c_{0}=\frac{y_{0}}{g(0, y(0), \underbrace{0, \ldots, 0}_{n}}$ and $c_{1}=\frac{y_{1}}{g\left(1, y(1), I^{\alpha} y(1), \ldots, I^{\alpha_{n}} y(1)\right)}$ $-I^{\alpha} x(1)-\frac{y_{0}}{g(0, y(0), \underbrace{0, \ldots, 0}_{n}}$. This completes the proof. 
$y \in \mathcal{Y}=C(J, \mathbb{R})$ is solution for the problem (1) whenever it satisfies the boundary conditions and there exists a function $v \in S_{G, y}$ such that

$$
\begin{aligned}
y(s) & =g\left(s, y(s), I^{\alpha_{1}} y(s), \ldots, I^{\alpha_{n}} y(s)\right) \\
& \times\left[I^{\alpha} v(s)+\frac{(1-s) y_{0}}{g(0, y(0), \underbrace{0, \ldots, 0}_{n})}+\frac{s y_{1}}{f\left(1, y(1), I^{\alpha_{1}} y(1), \ldots, I^{\alpha_{n}} y(1)\right)}-s I^{\alpha} v(1)\right] .
\end{aligned}
$$

where $S_{G, y}=\left\{v \in L^{1}[0,1]: v(s) \in G\left(s, y(s), I^{\beta_{1}} y(s), \ldots, I^{\beta_{k}} y(s)\right)\right.$ for almost all $\left.s \in J\right\}$.

Theorem 7 Let $G: J \times \mathbb{R}^{k+1} \rightarrow \mathcal{P}_{c p, c v}(\mathbb{R})$ be a Caratheodory multifunction, $g: J \times \mathbb{R}^{n+1} \rightarrow \mathbb{R}-\{0\}$ is a continuous and bounded function with bound $K$ and there exist continuous functions $p, m: J \rightarrow(0, \infty)$ such that $\left\|G\left(s, y_{1}, y_{2}, \ldots, y_{k}\right)\right\| \leq m(s)$ and $\left|g\left(s, y_{1}, y_{2}, \ldots, y_{n+1}\right)-g\left(s, x_{1}, x_{2}, \ldots, x_{n+1}\right)\right| \leq p(s) \sum_{i=1}^{n+1}\left|y_{i}-x_{i}\right|$ for all $s \in J$. If

$$
\begin{aligned}
& \|p\|_{\infty}\left(1+\frac{1}{\Gamma\left(\alpha_{1}+1\right)}+\frac{1}{\Gamma\left(\alpha_{2}+1\right)}+\cdots+\frac{1}{\Gamma\left(\alpha_{n}+1\right)}\right)\left(\frac{2\|m\|_{\infty}}{\Gamma(\alpha+1)}+\left|\frac{y_{0}}{g(0, y(0), \underbrace{0, \ldots, 0}_{n})}\right|\right. \\
& \left.+\left|\frac{y_{1}}{g\left(1, y(1), I^{\alpha_{1}} y(1), \ldots, I^{\alpha_{n}} y(1)\right)}\right|\right)<1,
\end{aligned}
$$

then the problem (1) has a solution.

Proof Define $S=\{y \in \mathcal{Y}:\|y\| \leq L\}$, where

$$
L=K\left(\frac{2\|m\|_{\infty}}{\Gamma(\alpha+1)}+\left|\frac{y_{0}}{g(0, y(0), \underbrace{0, \ldots, 0}_{n})}\right|+\left|\frac{y_{1}}{g\left(1, y(1), I^{\alpha_{1}} y(1), \ldots, I^{\alpha_{n}} y(1)\right)}\right|\right) .
$$

clearly $S$ is a closed, bounded and convex subset of the Banach algebra space $\mathcal{Y}$. Now, consider the multivalued operators $\mathcal{A}, \mathcal{B}: S \rightarrow \mathcal{P}(\mathcal{Y})$ by

$$
\mathcal{A} y(s)=\left\{g\left(s, y(s), I^{\alpha_{1}} y(s), \ldots, I^{\alpha_{n}} y(s)\right)\right\}
$$

and

$$
\begin{aligned}
\mathcal{B} y(s) & =\left\{u \in \mathcal{Y}: \text { there exists } v \in S_{G, y} \text { such that } u(s)=I^{\alpha} v(s)+\frac{(1-s) y_{0}}{g(0, y(0), \underbrace{0, \ldots, 0}_{n})}\right. \\
& \left.+\frac{s y_{1}}{g\left(1, y(1), I^{\alpha_{1}} y(1), \ldots, I^{\alpha_{n}} y(1)\right)}-s I^{\alpha} v(1) \quad \text { for all } s \in J\right\} .
\end{aligned}
$$

Thus, the problem (1) is tantamount to the problem $y \in \mathcal{A}(y) \mathcal{B}(y)$. We prove that the multifunctions $\mathcal{A}$ and $\mathcal{B}$ well-defined the conditions of Theorem 3. Note that, the operator $\mathcal{B}=\theta \circ S_{G}$, where $\theta$ is the continuous linear operator on $L^{1}(J, \mathbb{R})$ into $\mathcal{Y}$ defined by 


$$
\theta v(s)=I^{\alpha} v(s)+\frac{(1-s) y_{0}}{g(0, y(0), \underbrace{0, \ldots, 0}_{n})}+\frac{s y_{1}}{g\left(1, y(1), I^{\alpha_{1}} y(1), \ldots, I^{\alpha_{n}} y(1)\right)}-s I^{\alpha} v(1) .
$$

Let $y \in S$ be arbitrary and $\left\{v_{n}\right\}$ a sequence in $S_{G, y}$. Then, $v_{n}(s) \in G\left(s, y(s), I^{\beta_{1}} y(s), \ldots, I^{\beta_{k}} y(s)\right)$ for almost $s \in J$. Because $G\left(s, y(s), I^{\beta_{1}} y(s), \ldots, I^{\beta_{k}} y(s)\right)$ is compact for all $s \in J$, there exists a convergent subsequence of $\left\{v_{n}(s)\right\}$ (we show it again $\left.\left\{v_{n}(s)\right\}\right)$ to some $v \in S_{G, y}$. Since $\theta$ is continuous, $\theta v_{n}(s) \rightarrow \theta v(s)$ pointwise on $J$. Because we will show that the convergence is uniform, we have to prove that $\left\{\theta v_{n}\right\}$ is an equi-continuous sequence. Suppose that $\tau<s \in J$. So, we have

$$
\begin{aligned}
\left|\theta v_{n}(s)-\theta v_{n}(\tau)\right| \leq & \left.\frac{\|m\|_{\infty}\left(s^{\alpha}-\tau^{\alpha}\right)}{\Gamma(\alpha+1)}\right)+(s-\tau) \mid \frac{y_{0}}{g(0, y(0), \underbrace{0, \ldots, 0}_{n})} \\
& +\frac{y_{1}}{g\left(1, y(1), I^{\alpha_{1}} y(1), \ldots, I^{\alpha_{n}} y(1)\right)}-I^{\alpha} v_{n}(1) \mid .
\end{aligned}
$$

Hence the right hand of above inequalities tends to 0 as $s \rightarrow \tau$ and so the sequence $\left\{\theta v_{n}\right\}$ is equi-continuous. By using the Arzela-Ascoli theorem, it has a uniformly convergent subsequence. Thus, there is a subsequence of $\left\{v_{n}\right\}$ (we show it again by $\left\{v_{n}\right\}$ ) such that $\theta v_{n} \rightarrow \theta v$. Hence, $\theta v \in \theta\left(S_{G, y}\right)$. Thus, $\mathcal{B}=\theta\left(S_{G, y}\right)$ is compact for all $y \in S$. Now, we show that $\mathcal{B} y$ is convex for all $y \in S$. Let $y \in S$ and $u, u^{\prime} \in \mathcal{B} y$. Choose $v, v^{\prime} \in S_{G, y}$ such that

$$
\begin{gathered}
u(s)=I^{\alpha} v(s)+\frac{(1-s) y_{0}}{g(0, y(0), \underbrace{0, \ldots, 0}_{n})}+\frac{s y_{1}}{g\left(1, y(1), I^{\alpha_{1}} y(1), \ldots, I^{\alpha_{n}} y(1)\right)}-s I^{\alpha} v(1), \\
u^{\prime}(s)=I^{\alpha} v^{\prime}(s)+\frac{(1-s) y_{0}}{g(0, y(0), \underbrace{0, \ldots, 0}_{n})}+\frac{s y_{1}}{g\left(1, y(1), I^{\alpha_{1}} y(1), \ldots, I^{\alpha_{n}} y(1)\right)}-s I^{\alpha} v^{\prime}(1)
\end{gathered}
$$

for almost all $s \in J$. Let $0 \leq \lambda \leq 1$. Then, we have

$$
\begin{aligned}
\lambda u(s)+(1-\lambda) u^{\prime}(s)= & \frac{1}{\Gamma(\alpha)} \int_{0}^{s}(s-t)^{\alpha-1}\left[\lambda \nu(t)+(1-\lambda) v^{\prime}(t)\right] d s+\frac{(1-s) y_{0}}{g(0, y(0), \underbrace{0, \ldots, 0}_{n})} \\
& +\frac{s y_{1}}{g\left(1, y(1), I^{\alpha} y(1), \ldots, I^{\alpha_{n}} y(1)\right)} \\
& -\frac{s}{\Gamma(\alpha)} \int_{0}^{1}(1-t)^{\alpha-1}\left[\lambda v(t)+(1-\lambda) v^{\prime}(t)\right] d t .
\end{aligned}
$$

Since $G$ is convex valued, $\lambda u+(1-\lambda) u^{\prime} \in \mathcal{B} y$. Cleary, $\mathcal{A}$ is bounded, closed and convex valued. We prove that $\mathcal{A} y \mathcal{B} y$ is a convex subset of $S$ for all $y \in S$. Suppose that $y \in S$ and $u, u^{\prime} \in \mathcal{A} y \mathcal{B} y$. Choose $v, v^{\prime} \in S_{G, y}$ such that 


$$
\begin{gathered}
u(s)=g\left(s, y(s), I^{\alpha_{1}} y(s), \ldots, I^{\alpha_{n}} y(s)\right)\left[I^{\alpha} v(s)+\frac{(1-s) y_{0}}{g(0, y(0), \underbrace{0, \ldots, 0}_{n})}+\frac{s y_{1}}{g\left(1, y(1), I^{\alpha_{1}} y(1), \ldots, I^{\alpha_{n}} y(1)\right)}-s I^{\alpha} v(1)\right], \\
u^{\prime}(s)=g\left(s, y(s), I^{\alpha_{1}} y(s), \ldots, I^{\alpha_{n}} y(s)\right)\left[I^{\alpha} v^{\prime}(s)+\frac{(1-s) y_{0}}{g(0, y(0), \underbrace{0, \ldots, 0}_{n})}+\frac{s y_{1}}{g\left(1, s(1), I^{\alpha_{1}} s(1), \ldots, I^{\alpha_{n}} y(1)\right)}-s I^{\alpha} v^{\prime}(1)\right]
\end{gathered}
$$

for almost all $s \in J$. Hence, we get

$$
\begin{aligned}
\lambda u(s)+(1-\lambda) u^{\prime}(s)= & g\left(s, y(s), I^{\alpha_{1}} y(s), \ldots, I^{\alpha_{n}} y(s)\right)\left[\frac{1}{\Gamma(\alpha)} \int_{0}^{s}(s-t)^{\alpha-1}\left[\lambda v(t)+(1-\lambda) v^{\prime}(t)\right] d t\right. \\
& +\frac{(1-s) y_{0}}{g(0, y(0), \underbrace{0, \ldots, 0}_{n})}+\frac{s y_{1}}{g\left(1, y(1), I^{\alpha_{1}} y(1), \ldots, I^{\alpha_{n}} y(1)\right)} \\
& -\frac{s}{\Gamma(\alpha)} \int_{0}^{1}(1-t)^{\alpha-1}\left[\lambda v(t)+(1-\lambda) v^{\prime}(t)\right] d t .
\end{aligned}
$$

Since $G$ is convex valued, $\lambda u+(1-\lambda) u^{\prime} \in \mathcal{A} y \mathcal{B} y$. So, $\mathcal{A} y \mathcal{B} y$ is convex subset of $\mathcal{Y}$ for all $y \in \mathcal{Y}$. But, we have

$$
\begin{aligned}
|u(s)|= & \mid g\left(s, y(s), I^{\alpha_{1}} y(s), \ldots, I^{\alpha_{n}} y(s)\right)\left[I^{\alpha} v(s)+\frac{(1-s) y_{0}}{g(0, y(0), \underbrace{0, \ldots, 0}_{n})}+\frac{s y_{1}}{g\left(1, y(1), I^{\alpha_{1}} y(1), \ldots, I^{\alpha_{n}} y(1)\right)}\right. \\
& \left.-s I^{\alpha} v(1)\right] \mid \leq K\left(\frac{2\|m\|_{\infty}}{\Gamma(\alpha+1)}+\left|\frac{y_{0}}{g(0, y(0), \underbrace{0, \ldots, 0}_{n})}\right|+\left|\frac{y_{1}}{g\left(1, y(1), I^{\alpha_{1}} y(1), \ldots, I^{\alpha_{n}} y(1)\right)}\right|\right)=L
\end{aligned}
$$

for all $s \in J$. So, $u \in S$ and $\mathcal{A} y \mathcal{B} y$ is a convex subset of $S$ for all $y \in S$. Here, We show that operator $\mathcal{B}$ is compact. For showing this, it is enough to prove that $\mathcal{B}(S)$ is uniformly bounded and equi-continuous. Let $u \in \mathcal{B}(S)$. Choose $v \in S_{G, y}$ such that $u(s)=g\left(s, y(s), I^{\alpha_{1}} y(s), \ldots, I^{\alpha_{n}} y(s)\right)\left[I^{\alpha} v(s)+\frac{(1-s) y_{0}}{g(0, y(0), \underbrace{0, \ldots, 0}_{n})}+\frac{s y_{1}}{g\left(1, y(1), I^{\alpha_{1}} y(1), \ldots, I^{\alpha_{n}} y(1)\right)}-s I^{\alpha} v(1)\right]$ for some $y \in S$. Hence,

$$
|u(s)| \leq\left(\frac{2\|m\|_{\infty}}{\Gamma(\alpha+1)}+\left|\frac{y_{0}}{g(0, y(0), \underbrace{0, \ldots, 0}_{n})}\right|+\left|\frac{y_{1}}{g\left(1, y(1), I^{\alpha_{1}} y(1), \ldots, I^{\alpha_{n}} y(1)\right)}\right|\right)
$$

and so $\|u\|_{\infty}=\max _{s \in J}|u(s)| \leq\left(\frac{2\|m\|_{\infty}}{\Gamma(\alpha+1)}+\left|\frac{y_{0}}{g(0, y(0), \underbrace{0, \ldots, 0}_{n})}\right|+\left|\frac{y_{1}}{g\left(1, y(1), I^{\alpha} 1 y(1), \ldots, I^{\left.\alpha_{n} y(1)\right)}\right.}\right|\right)$. In this part, prove that $\mathcal{B}$ maps $S$ to equi-continuous subsets of $\mathcal{Y}$. Suppose that $s, \tau \in J$ with $\tau<s, y \in S$ and $u \in \mathcal{B} y$. Choose $v \in S_{G, y}$ such that $u(s)=I^{\alpha} v(s)+\frac{(1-s) y_{0}}{g(0, y(0), \underbrace{0, \ldots, 0}_{n})}+\frac{s y_{1}}{g\left(1, y(1), I^{\alpha} 1 y(1), \ldots, I^{\alpha_{n}} y(1)\right)}-s I^{\alpha} v(1)$. Then, we have 


$$
\begin{aligned}
|u(s)-u(\tau)| \leq & \left(\frac{\|m\|_{\infty}\left(s^{\alpha}-\tau^{\alpha}\right)}{\Gamma(\alpha+1)}\right)+(s-\tau) \mid \frac{y_{0}}{g(0, y(0), \underbrace{0, \ldots, 0}_{n})} \\
& +\frac{y_{1}}{g\left(1, y(1), I^{\alpha_{1}} y(1), \ldots, I^{\alpha_{n}} y(1)\right)}-I^{\alpha} v(1) \mid .
\end{aligned}
$$

So the right side of inequality towards to 0 as $s \rightarrow \tau$. Hence by using the Arzela-Ascoli theorem, $\mathcal{B}$ is compact. Here, we show that $\mathcal{B}$ has a closed graph. Suppose that $y_{n} \in S$ and $u_{n} \in \mathcal{B} y_{n}$ for all $n$ such that $y_{n} \rightarrow y^{\prime}$ and $u_{n} \rightarrow u^{\prime}$. We show that $u^{\prime} \in \mathcal{B} y^{\prime}$. For each natural number $n$, choose $v_{n} \in S_{G, y_{n}}$ such that

$$
u_{n}(s)=I^{\alpha} v_{n}(s)+\frac{(1-s) y_{0}}{g(0, y(0), \underbrace{0, \ldots, 0}_{n})}+\frac{s y_{1}}{g\left(1, y(1), I^{\alpha_{1}} y(1), \ldots, I^{\alpha_{n}} y(1)\right)}-s I^{\alpha} v_{n}(1)
$$

for all $s \in J$. Again, consider the continuous linear operator $\theta: L^{1}(J, \mathbb{R}) \rightarrow \mathcal{Y}$ such that

$\theta(v)(s)=u(s)=I^{\alpha} v(s)+\frac{(1-s) y_{0}}{g(0, y(0), \underbrace{0, \ldots, 0}_{n})}+\frac{s y_{1}}{g\left(1, y(1), I^{\alpha_{1}} y(1), \ldots, I^{\alpha_{n}} y(1)\right)}-s I^{\alpha} v(1)$.

By using Lemma 2, $\theta 0 S_{G}$ is a closed graph operator. Since $y_{n} \rightarrow y^{\prime}$ and $u_{n} \in \theta\left(S_{G, y_{n}}\right)$ for all $n$, there is $v^{\prime} \in S_{G, y^{\prime}}$ such that

$$
u^{\prime}(s)=I^{\alpha} v^{\prime}(s)+\frac{(1-s) y_{0}}{g(0, y(0), \underbrace{, \ldots, 0}_{n})}+\frac{s y_{1}}{g\left(1, y(1), I^{\alpha_{1}} y(1), \ldots, I^{\alpha_{n}} y(1)\right)}-s I^{\alpha} v^{\prime}(1) .
$$

Hence, $u^{\prime} \in \mathcal{B} y^{\prime}$. This implies that, $\mathcal{B}$ has a closed graph and thus the operator $\mathcal{B}$ is upper semi-continuous. Now, we show that $\mathcal{A}$ is a contractive multifunction. Note that,

$$
\begin{aligned}
H(\mathcal{A} y, \mathcal{A} x) & =\|\mathcal{A} y-\mathcal{A} x\|=\max _{s \in J}\left|g\left(s, y(s), I^{\alpha_{1}} y(s), \ldots, I^{\alpha_{n}} y(s)\right)-g\left(s, x(s), I^{\alpha_{1}} x(s), \ldots, I^{\alpha_{n}} x(s)\right)\right| \\
& \leq \max _{s \in J}(|p(s) \| y(s)-x(s)|)\left(1+\frac{1}{\Gamma\left(\alpha_{1}+1\right)}+\frac{1}{\Gamma\left(\alpha_{2}+1\right)}+\cdots+\frac{1}{\Gamma\left(\alpha_{n}+1\right)}\right) \\
& =\|p\|_{\infty}\left(1+\frac{1}{\Gamma\left(\alpha_{1}+1\right)}+\frac{1}{\Gamma\left(\alpha_{2}+1\right)}+\cdots+\frac{1}{\Gamma\left(\alpha_{n}+1\right)}\right)\|y-x\|_{\infty}
\end{aligned}
$$

for all $x, y \in \mathcal{Y}$. So, $\mathcal{A}$ and $\mathcal{B}$ satisfy the conditions of Theorem 3 and thus the operator inclusions $y \in \mathcal{A} y \mathcal{A} y$ has a solution in $S$. Therefore, the problem (1) has a solution.

To illustrate our main results, we present the following example:

Example 1 Here, we investigation the problem

$$
{ }^{c} D^{\frac{1}{2}}\left(\frac{y(s)}{\frac{(s+1)^{2}}{60} \sin y(s)+\frac{\left|I^{\sqrt{2}} y(s)\right|}{1+\left|I^{\sqrt{2}} y(s)\right|}+3}\right) \in\left[-1, s^{2} \sin y(s)+\cos \left(I^{\frac{1}{4}} y(s)\right)+1\right]
$$

with the boundary conditions $y(0)=\frac{\pi}{2}$ and $y(1)=0$. Put $\alpha=\frac{1}{2}, \alpha_{1}=\sqrt{2}$, $\beta_{1}=\frac{1}{4}, \quad n=k=1, \quad y_{0}=\frac{\pi}{2}, \quad y_{1}=0, \quad g(s, y, x)=\frac{(s+1)^{2}}{60} \sin y+\frac{|x|}{1+|x|}+3$, 
$G(s, y, x)=\left[-1, s^{2} \sin y+\cos x+1\right], m(s)=s^{2}+2$ and $p(s)=\frac{(s+1)^{2}}{60}$ for $s \in[0,1]$. Note that, $\|G(s, y, x)\| \leq s^{2}+2$,

$$
\left|g(s, y, x)-g\left(s, y^{\prime}, x^{\prime}\right)\right| \leq \frac{(s+1)^{2}}{60}\left(\left|y-y^{\prime}\right|+\left|x-x^{\prime}\right|\right)
$$

and $\|p\|_{\infty}\left(1+\frac{1}{\Gamma\left(\alpha_{1}+1\right)}\right)\left(\frac{2\|m\|_{\infty}}{\Gamma(\alpha+1)}+\left|\frac{y_{0}}{g(0, y(0), 0)}\right|+\left|\frac{y_{1}}{g\left(1, y(1), I^{\alpha} y(1)\right)}\right|\right)=0.8784698182<1$. By using the Theorem 7 , the problem $(*)$ has a solution.

Now, we review existence and dimension of the solution set of the fractional differential inclusion problem

$$
{ }^{c} D^{\alpha} y(s) \in G\left(s, y(s),(\phi y)(s),(\psi y)(s),{ }^{c} D^{\beta_{1}} y(s), \ldots,{ }^{c} D^{\beta_{k}} y(s), I^{\gamma_{1}} y(s), \ldots, I^{\gamma_{k}} y(s)\right)
$$

with boundary condition $y(0)+\sum_{i=1}^{k}{ }^{c} D^{\beta_{i}} y(1)+\sum_{i=1}^{k} I^{\gamma_{i}} y(1)=0$, where $0<\beta_{i}<\alpha \leq 1$ and $0<\gamma_{i}<1$ for $i=1, \ldots, k, G: J \times \mathbb{R}^{2 k+3} \rightarrow \mathcal{P}(\mathbb{R})$ is a multifunction via some properties, $\gamma, \lambda: J \times J \rightarrow[0, \infty)$ are two mappings with the properties $\sup _{s \in J}\left|\int_{0}^{s} \lambda(s, t) d s\right|<\infty$ and $\sup _{s \in J}\left|\int_{0}^{s} \gamma(s, t) d s\right|<\infty$ and the functions $\phi$ and $\psi$ are defined by $(\phi y)(s)=\int_{0}^{s} \gamma(s, t) y(t) d t$ and $(\psi y)(s)=\int_{0}^{s} \lambda(s, t) y(t) d t$.

Lemma 8 Suppose that $v \in C(J, \mathbb{R}), \alpha \in(0,1]$ and $\beta_{i}, \gamma_{i} \in(0,1)$ with $\alpha-\beta_{i}>0$ for $1 \leq i \leq k$. Then solution of the problem ${ }^{c} D^{\alpha} y(s)=v(s)$ with the boundary condition $y(0)+\sum_{i=1}^{k}{ }^{c} D^{\beta_{i}} y(1)+\sum_{i=1}^{k} I^{\gamma_{i}} y(1)=0$ is

$$
\begin{aligned}
y(s)= & \frac{1}{\Gamma(\alpha)} \int_{0}^{s}(s-t)^{\alpha-1} v(t) d t+\frac{-1}{1+\sum_{i=1}^{k} \frac{1}{\Gamma\left(\gamma_{i}+1\right)}} \sum_{i=1}^{k}\left(\frac{1}{\Gamma\left(\alpha-\beta_{i}\right)} \int_{0}^{1}(1-t)^{\alpha-\beta_{i}-1} v(t) d t\right. \\
& \left.+\frac{1}{\Gamma\left(\alpha+\gamma_{i}\right)} \int_{0}^{1}(1-t)^{\alpha+\gamma_{i}-1} v(t) d t\right) .
\end{aligned}
$$

Proof The general solution of the problem ${ }^{c} D^{\alpha} y(s)=v(s)$ is formed by

$$
y(s)=I^{\alpha} v(s)+c_{0}=\frac{1}{\Gamma(\alpha)} \int_{0}^{s}(s-t)^{\alpha-1} v(t) d t+c_{0},
$$

where $c_{0}$ is arbitrary constant and $t \in J$ (Podlubny 1999). Thus, we obtain

$$
{ }^{c} D^{\beta_{i}} y(s)=I^{\alpha-\beta_{i}} v(s)=\frac{1}{\Gamma\left(\alpha-\beta_{i}\right)} \int_{0} s(s-t)^{\alpha-\beta_{i}-1} v(t) d t
$$

and

$$
I^{\gamma_{i}} y(s)=I^{\alpha+\gamma_{i}} v(s)+\frac{c_{0} s^{\gamma_{i}}}{\Gamma\left(\gamma_{i}+1\right)}=\frac{1}{\Gamma\left(\alpha+\gamma_{i}\right)} \int_{0}^{s}(s-t)^{\alpha+\gamma_{i}-1} v(t) d t+\frac{c_{0} s^{\gamma_{i}}}{\Gamma\left(\gamma_{i}+1\right)}
$$

for all $1 \leq i \leq k$. By using the boundary condition, we get

$$
\begin{aligned}
c_{0}= & \frac{-1}{1+\sum_{i=1}^{k} \frac{1}{\Gamma\left(\gamma_{i}+1\right)}} \sum_{i=1}^{k}\left(\frac{1}{\Gamma\left(\alpha-\beta_{i}\right)} \int_{0}^{1}(1-t)^{\alpha-\beta_{i}-1} v(t) d t\right. \\
& \left.+\frac{1}{\Gamma\left(\alpha+\gamma_{i}\right)} \int_{0}^{1}(1-t)^{\alpha+\gamma_{i}-1} v(t) d t\right) .
\end{aligned}
$$


Hence,

$$
\begin{aligned}
y(s)= & \frac{1}{\Gamma(\alpha)} \int_{0}^{s}(s-t)^{\alpha-1} v(t) d t+\frac{-1}{1+\sum_{i=1}^{k} \frac{1}{\Gamma\left(\gamma_{i}+1\right)}} \sum_{i=1}^{k}\left(\frac{1}{\Gamma\left(\alpha-\beta_{i}\right)} \int_{0}^{1}(1-t)^{\alpha-\beta_{i}-1} v(t) d t\right. \\
& \left.+\frac{1}{\Gamma\left(\alpha+\gamma_{i}\right)} \int_{0}^{1}(1-t)^{\alpha+\gamma_{i}-1} v(t) d t\right) .
\end{aligned}
$$

This completes the proof.

An element $y \in C(J, \mathbb{R})$ is a solution for the problem (2) whenever it satisfies the boundary condition and there is a function $v \in L^{1}(J)$ such that

$$
v(s) \in G\left(s, y(s),(\phi y)(s),(\psi y)(s),{ }^{c} D^{\beta_{1}} y(s), \ldots,{ }^{c} D^{\beta_{k}} y(s), I^{\gamma_{1}} y(s), \ldots, I^{\gamma_{k}} y(s)\right)
$$

for almost all $s \in J$ and

$$
\begin{aligned}
y(s)= & \frac{1}{\Gamma(\alpha)} \int_{0}^{s}(s-t)^{\alpha-1} v(t) d t+\frac{-1}{1+\sum_{i=1}^{k} \frac{1}{\Gamma\left(\gamma_{i}+1\right)}} \sum_{i=1}^{k}\left(\frac{1}{\Gamma\left(\alpha-\beta_{i}\right)} \int_{0}^{1}(1-t)^{\alpha-\beta_{i}-1} v(t) d t\right. \\
& \left.+\frac{1}{\Gamma\left(\alpha+\gamma_{i}\right)} \int_{0}^{1}(1-t)^{\alpha+\gamma_{i}-1} v(t) d t\right) .
\end{aligned}
$$

Put $\mathcal{Y}=\left\{y: y,{ }^{c} D^{\beta_{i}} y \in C(J, \mathbb{R})\right.$ for each $\left.i \in\{1, \ldots, k\}\right\}$ with the norm

$$
\|y\|=\sup _{s \in J}|y(s)|+\sum_{i=1}^{k} \sup _{s \in J}\left|{ }^{c} D^{\beta_{i}} y(s)\right| \text {. }
$$

$(\mathcal{Y},\|\|$.$) is a Banach space (Su 2009). Define selection set of G$ at $y \in \mathcal{Y}$ by

$$
\begin{aligned}
S_{G, y}:= & \left\{v \in L^{1}(J, \mathbb{R}): v(s) \in G\left(s, y(s),(\phi y)(s),(\psi y)(s),{ }^{c} D^{\beta_{1}} y(s), \ldots,{ }^{c} D^{\beta_{k}} y(s),\right.\right. \\
& \left.\left.I^{\gamma_{1}} y(s), \ldots, I^{\gamma_{k}} y(s)\right) \text { for almost all } s \in J\right\} .
\end{aligned}
$$

Theorem 9 Suppose that $m \in L^{1}\left(J, \mathbb{R}^{+}\right), l=\left(\Lambda_{1}+\sum_{i=1}^{k} \Lambda_{2}^{i}\right)<1$, where

$$
\Lambda_{1}=\|m\|_{1}\left(1+\lambda_{0}+\gamma_{0}+\sum_{i=1}^{k} \frac{1}{\Gamma\left(\gamma_{i}+1\right)}\right)\left(\frac{1}{\Gamma(\alpha)}+\frac{1}{1+\sum_{i=1}^{k} \frac{1}{\Gamma\left(\gamma_{i}+1\right)}} \sum_{i=1}^{k} \frac{1}{\Gamma\left(\alpha-\beta_{i}\right)}+\frac{1}{\Gamma\left(\alpha+\gamma_{i}\right)}\right)
$$

$\Lambda_{2}^{i}=\|m\|_{1}\left(1+\lambda_{0}+\gamma_{0}+\sum_{i=1}^{k} \frac{1}{\Gamma\left(\gamma_{i}+1\right)}\right) \frac{1}{\Gamma\left(\alpha-\beta_{i}\right)} \quad$ for $\quad i=1, \ldots, k, \quad F: J \times \mathbb{R}^{2 k+3}$ $\rightarrow \mathcal{P}_{c v, c p}(\mathbb{R})$ is a multifunction such that the map $s \vdash G\left(s, y_{1}, y_{2}, \ldots, 2 k+3\right)$ is measurable,

$\left\|G\left(s, y_{1}, y_{2}, \ldots, 2 k+3\right)\right\|=\sup \left\{|v|: v \in G\left(s, y_{1}, y_{2}, \ldots, 2 k+3\right)\right\} \leq m(s)$

and $\left.H\left(G\left(s, y_{1}, y_{2}, \ldots, y_{2 k+3}\right)\right), G\left(s, x_{1}, x_{2}, \ldots, x_{2 k+3}\right)\right) \leq m(s) \sum_{i=1}^{2 k+3}\left(\left|y_{i}-x_{i}\right|\right)$ for almost all $s \in J$ and $\in x_{1}, x_{2}, \ldots, x_{2 k+3}, y_{1}, y_{2}, \ldots, y_{2 k+3} \in \mathbb{R}$. Then the inclusion problem (2) has a solution.

Proof Note that, the multivalued map 


$$
s \vdash G\left(s, y(s),(\phi y)(s),(\psi y)(s),{ }^{c} D^{\beta_{1}} y(s), \ldots,{ }^{c} D^{\beta_{k}} y(s), I^{\gamma_{1}} y(s), \ldots, I^{\gamma_{k}} y(s)\right)
$$

is measurable and closed valued for all $y \in \mathcal{Y}$. Hence, it has a measurable selection and so the set $S_{G, y}$ is nonempty. Now, consider the operator $\Upsilon: \mathcal{Y} \rightarrow 2^{\mathcal{Y}}$ defined by

$$
\Upsilon(y)=\left\{\xi \in X: \text { there is } v \in S_{G, y}: \xi(s)=v(s) \text { for all } s \in J\right\},
$$

where

$$
\begin{aligned}
v(s)= & \frac{1}{\Gamma(\alpha)} \int_{0}^{s}(s-t)^{\alpha-1} v(t) d t+\frac{-1}{1+\sum_{i=1}^{k} \frac{1}{\Gamma\left(\gamma_{i}+1\right)}} \sum_{i=1}^{k}\left(\frac{1}{\Gamma\left(\alpha-\beta_{i}\right)} \int_{0}^{1}(1-t)^{\alpha-\beta_{i}-1} v(t) d t\right. \\
& \left.+\frac{1}{\Gamma\left(\alpha+\gamma_{i}\right)} \int_{0}^{1}(1-t)^{\alpha+\gamma_{i}-1} v(t) d t\right)
\end{aligned}
$$

for all $s \in J$. Here, we prove that $\Upsilon(y)$ is a closed subset of $\mathcal{Y}$ for each $y \in \mathcal{Y}$. Let $y \in \mathcal{Y}$ and $\left\{u_{n}\right\}_{n \geq 1}$ be a sequence in $\Upsilon(y)$ with $u_{n} \rightarrow u$. For each $n$, choose $v_{n} \in S_{G, y}$ such that

$$
\begin{aligned}
u_{n}(s)= & \frac{1}{\Gamma(\alpha)} \int_{0}^{s}(s-t)^{\alpha-1} v_{n}(t) d t+\frac{-1}{1+\sum_{i=1}^{k} \frac{1}{\Gamma\left(\gamma_{i}+1\right)}} \sum_{i=1}^{k}\left(\frac{1}{\Gamma\left(\alpha-\beta_{i}\right)} \int_{0}^{1}(1-t)^{\alpha-\beta_{i}-1} v_{n}(t) d t\right. \\
& \left.+\frac{1}{\Gamma\left(\alpha+\gamma_{i}\right)} \int_{0}^{1}(1-t)^{\alpha+\gamma_{i}-1} v_{n}(t) d t\right)
\end{aligned}
$$

for almost all $s \in J$. because $G$ has compact values, $\left\{v_{n}\right\}_{n \geq 1}$ has a subsequence which converges to a $v \in L^{1}(J, \mathbb{R})$. We write it again by $\left\{v_{n}\right\}_{n \geq 1}$. Clearly $v \in S_{G, y}$ and

$$
\begin{aligned}
u_{n}(s) \rightarrow u(s)= & \frac{1}{\Gamma(\alpha)} \int_{0}^{s}(s-t)^{\alpha-1} v(t) d t+\frac{-1}{1+\sum_{i=1}^{k} \frac{1}{\Gamma\left(\gamma_{i}+1\right)}} \sum_{i=1}^{k}\left(\frac{1}{\Gamma\left(\alpha-\beta_{i}\right)} \int_{0}^{1}(1-t)^{\alpha-\beta_{i}-1} v(t) d t\right. \\
& \left.+\frac{1}{\Gamma\left(\alpha+\gamma_{i}\right)} \int_{0}^{1}(1-t)^{\alpha+\gamma_{i}-1} v(t) d t\right)
\end{aligned}
$$

for all $s \in J$. This implies that $u \in \Upsilon(x)$. Thus, the multifunction $\Upsilon$ has closed values. Now, we show that $\Upsilon$ is a contractive multifunction with constant $l=\left(\Lambda_{1}+\sum_{i=1}^{k} \Lambda_{2}^{i}\right)<1$. Suppose that $x, y \in \mathcal{Y}$ and $h_{1} \in \Upsilon(x)$. Consider $v_{1} \in S_{G, x}$ such that

$$
\begin{aligned}
h_{1}(s)= & \frac{1}{\Gamma(\alpha)} \int_{0}^{s}(s-t)^{\alpha-1} v_{1}(t) d t+\frac{-1}{1+\sum_{i=1}^{k} \frac{1}{\Gamma\left(\gamma_{i}+1\right)}} \sum_{i=1}^{k}\left(\frac{1}{\Gamma\left(\alpha-\beta_{i}\right)} \int_{0}^{1}(1-t)^{\alpha-\beta_{i}-1} v_{1}(t) d t\right. \\
& \left.+\frac{1}{\Gamma\left(\alpha+\gamma_{i}\right)} \int_{0}^{1}(1-t)^{\alpha+\gamma_{i}-1} v_{1}(t) d t\right)
\end{aligned}
$$

for almost all $s \in J$. Since

$$
\begin{aligned}
& H\left(G\left(s, y(s),(\phi y)(s),(\psi y)(s),{ }^{c} D^{\beta_{1}} y(s), \ldots,{ }^{c} D^{\beta_{k}} y(s), I^{\gamma_{1}} y(s), \ldots, I^{\gamma_{k}} y(s)\right),\right. \\
& \left.G\left(s, x(s),(\phi x)(s),(\psi x)(s),{ }^{c} D^{\beta_{1}} x(s), \ldots,{ }^{c} D^{\beta_{k}} x(s), I^{\gamma_{1}} x(s), \ldots, I^{\gamma_{k}} x(s)\right)\right) \\
& \leq m(t)(|y(s)-x(s)|+|(\phi y)(s)-(\phi x)(s)|+|(\psi y)(s)-(\psi x)(s)| \\
& \left.\quad+\sum_{i=1}^{k}\left|{ }^{c} D^{\beta_{i}} y(s)-{ }^{c} D^{\beta_{i}} x(s)\right|+\sum_{i=1}^{k}\left|I^{\gamma_{i}} y(s)-I^{\gamma_{i}} x(s)\right|\right)
\end{aligned}
$$


for almost all $s \in J$, there is

$$
v \in\left(G\left(s, y(s),(\phi y)(s),(\psi y)(s),{ }^{c} D^{\beta_{1}} y(s), \ldots,{ }^{c} D^{\beta_{k}} y(s), I^{\gamma_{1}} y(s), \ldots, I^{\gamma_{k}} y(s)\right)\right)
$$

such that

$$
\begin{aligned}
\left|v_{1}(s)-v\right| \leq & m(s)(|y(s)-x(s)|+|(\phi y)(s)-(\phi x)(s)|+|(\psi y)(s)-(\psi x)(s)| \\
& \left.+\sum_{i=1}^{k}\left|{ }^{c} D^{\beta_{i}} y(s)-{ }^{c} D^{\beta_{i}} x(s)\right|+\sum_{i=1}^{k}\left|I^{\gamma_{i}} y(s)-I^{\gamma_{i}} x(s)\right|\right)
\end{aligned}
$$

for almost all $s \in J$. Define the multifunction $\Delta: J \rightarrow 2^{\mathbb{R}}$ by

$$
\begin{aligned}
\Delta(s)= & \left\{v \in \mathbb{R}:\left|\nu_{1}(s)-v\right| \leq m(s)(|y(s)-x(s)|+|(\phi y)(s)-(\phi x)(s)|+|(\psi y)(s)-(\psi x)(s)|\right. \\
& \left.\left.+\sum_{i=1}^{k}\left|{ }^{c} D^{\beta_{i}} y(s)-{ }^{c} D^{\beta_{i}} x(s)\right|+\sum_{i=1}^{k}\left|I^{\gamma_{i}} y(s)-I^{\gamma_{i}} x(s)\right|\right) \quad \text { for almost all } s \in J\right\} .
\end{aligned}
$$

The multifunction

$$
\Delta(.) \bigcap\left(G\left(., y(.),(\phi y)(.),(\psi y)(.),{ }^{c} D^{\beta_{1}} y(.),{ }^{c} D^{\beta_{2}} y(.), \ldots,{ }^{c} D^{\beta_{k}} y(.), I^{\gamma_{1}} y(.), I^{\gamma_{2}} y(.), \ldots, I^{\gamma_{k}} y(.)\right)\right)
$$

is measurable. Thus, we can choose $v_{2} \in S_{G, y}$ such that

$$
\begin{aligned}
\left|v_{1}(s)-v_{2}(s)\right| \leq & m(s)(|y(s)-x(s)|+|(\phi y)(s)-(\phi x)(s)|+|(\psi y)(s)-(\psi x)(s)| \\
& \left.+\sum_{i=1}^{k}\left|{ }^{c} D^{\beta_{i}} y(s)-{ }^{c} D^{\beta_{i}} x(s)\right|+\sum_{i=1}^{k}\left|I^{\gamma_{i}} y(s)-I^{\gamma_{i}} x(s)\right|\right)
\end{aligned}
$$

for almost all $s \in J$. Now, define $h_{2} \in \Upsilon(y)$ by

$$
\begin{aligned}
h_{2}(s)= & \frac{1}{\Gamma(\alpha)} \int_{0}^{s}(s-t)^{\alpha-1} v_{2}(t) d t+\frac{-1}{1+\sum_{i=1}^{k} \frac{1}{\Gamma\left(\gamma_{i}+1\right)}} \sum_{i=1}^{k}\left(\frac{1}{\Gamma\left(\alpha-\beta_{i}\right)} \int_{0}^{1}(1-t)^{\alpha-\beta_{i}-1} v_{2}(t) d t\right. \\
& \left.+\frac{1}{\Gamma\left(\alpha+\gamma_{i}\right)} \int_{0}^{1}(1-t)^{\alpha+\gamma_{i}-1} v_{2}(t) d t\right) .
\end{aligned}
$$

So

$$
\begin{aligned}
\left|h_{1}(s)-h_{2}(s)\right| \leq & \frac{1}{\Gamma(\alpha)} \int_{0}^{s}(s-t)^{\alpha-1}\left|\nu_{1}(t)-v_{2}(t)\right| d t \\
& +\frac{1}{1+\sum_{i=1}^{k} \frac{1}{\Gamma\left(\gamma_{i}+1\right)}} \sum_{i=1}^{k}\left(\frac{1}{\Gamma\left(\alpha-\beta_{i}\right)} \int_{0}^{1}(1-t)^{\alpha-\beta_{i}-1}\left|v_{1}(t)-v_{2}(t)\right| d t\right. \\
& \left.+\frac{1}{\Gamma\left(\alpha+\gamma_{i}\right)} \int_{0}^{1}(1-t)^{\alpha+\gamma_{i}-1}\left|\nu_{1}(t)-v_{2}(t)\right| d t\right) \leq \Lambda_{1}\|y-x\|
\end{aligned}
$$

and $\left|{ }^{c} D^{\beta_{i}} h_{1}(s)-{ }^{c} D^{\beta_{i}} h_{2}(s)\right| \leq \frac{1}{\Gamma\left(\alpha-\beta_{i}\right)} \int_{0}^{s}(s-t)^{\alpha-\beta_{i}-1}\left|v_{1}(t)-v_{2}(t)\right| \leq \Lambda_{2}^{i}\|x-y\|$ and so we get $\left\|h_{1}-h_{2}\right\| \leq\left(\Lambda_{1}+\sum_{i=1}^{k} \Lambda_{2}^{i}\right)\|x-y\|=l\|x-y\|$. This implies that the multifunction $N$ is a contraction via closed values. By using the well-known theorem of Covitz and Nadler, $N$ has a fixed point which is a solution for the inclusion problem (2). 
Lemma 10 Suppose that $m \in L^{1}\left(J, \mathbb{R}^{+}\right), G: J \times \mathbb{R}^{2 k+3} \rightarrow \mathcal{P}_{c v, c p}(\mathbb{R})$ is a multifunction such that the map $s \vdash G\left(s, y_{1}, y_{2}, \ldots, y_{2 k+3}\right)$ is measurable,

$$
\left\|G\left(s, y_{1}, y_{2}, \ldots, y_{2 k+3}\right)\right\|=\sup \left\{|v|: v \in G\left(s, y_{1}, y_{2}, \ldots, y_{2 k+3}\right)\right\} \leq m(s)
$$

for almost all $s \in J$ and $\in y_{1}, y_{2}, \ldots, y_{2 k+3} \in \mathbb{R}$ and $\Upsilon: \mathcal{Y} \rightarrow \mathcal{P}(\mathcal{Y})$ is defined by

$$
\Upsilon(y)=\left\{\xi \in \mathcal{Y}: \text { there is } v \in S_{G, y}: \xi(s)=\nu(s) \text { for all } s \in J\right\},
$$

where

$$
\begin{aligned}
v(s)= & \frac{1}{\Gamma(\alpha)} \int_{0}^{s}(s-t)^{\alpha-1} v(t) d t+\frac{-1}{1+\sum_{i=1}^{k} \frac{1}{\Gamma\left(\gamma_{i}+1\right)}} \sum_{i=1}^{k}\left(\frac{1}{\Gamma\left(\alpha-\beta_{i}\right)} \int_{0}^{1}(1-t)^{\alpha-\beta_{i}-1} v(t) d t\right. \\
& \left.+\frac{1}{\Gamma\left(\alpha+\gamma_{i}\right)} \int_{0}^{1}(1-t)^{\alpha+\gamma_{i}-1} v(t) d t\right) .
\end{aligned}
$$

Then, $\Upsilon(y) \in \mathcal{P}_{c p . c v}(\mathcal{Y})$ for all $y \in \mathcal{Y}$.

Proof Note that the operator $\Upsilon=\theta \circ S_{G}$, where $\theta$ is the continuous linear operator on $L^{1}(J, \mathbb{R})$ into $\mathcal{Y}$ which is defined by

$$
\begin{aligned}
\theta v(s)= & \frac{1}{\Gamma(\alpha)} \int_{0}^{s}(s-t)^{\alpha-1} v(t) d t+\frac{-1}{1+\sum_{i=1}^{k} \frac{1}{\Gamma\left(\gamma_{i}+1\right)}} \sum_{i=1}^{k}\left(\frac{1}{\Gamma\left(\alpha-\beta_{i}\right)} \int_{0}^{1}(1-t)^{\alpha-\beta_{i}-1} v(t) d t\right. \\
& \left.+\frac{1}{\Gamma\left(\alpha+\gamma_{i}\right)} \int_{0}^{1}(1-t)^{\alpha+\gamma_{i}-1} v(t) d t\right) .
\end{aligned}
$$

Suppose that $y \in \mathcal{Y}$ and $\left\{v_{n}\right\}$ is a sequence in $S_{G, y}$. so,

$$
v_{n}(s) \in G\left(s, y(s),(\phi y)(s),(\psi y)(s),{ }^{c} D^{\beta_{1}} y(s), \ldots,{ }^{c} D^{\beta_{k}} y(s), I^{\gamma_{1}} y(s), \ldots, I^{\gamma_{k}} y(s)\right)
$$

for almost $s \in J$. Since

$$
G\left(s, y(s),(\phi y)(s),(\psi y)(s),{ }^{c} D^{\beta_{1}} y(s), \ldots,{ }^{c} D^{\beta_{k}} y(s), I^{\gamma_{1}} y(s), \ldots, I^{\gamma_{k}} y(s)\right)
$$

is compact for all $s \in J$, there exists a convergent subsequence of $\left\{v_{n}(s)\right\}$ (we show it again by $\left.\left\{v_{n}(s)\right\}\right)$ which converges to some $v \in S_{G, y}$. Since $\theta$ is continuous, $\theta v_{n}(s) \rightarrow \theta v(s)$ pointwise on $J$. Because we show that the convergence is uniform, we must prove that $\left\{\theta v_{n}\right\}$ is an equi-continuous sequence. Let $\tau<s \in J$. Then

$$
\begin{array}{r}
\left|\theta v_{n}(s)-\theta v_{n}(\tau)\right|=\left|\frac{1}{\Gamma(\alpha)} \int_{0}^{s}(s-t)^{\alpha-1} v_{n}(t) d t-\frac{1}{\Gamma(\alpha)} \int_{0}^{\tau}(\tau-t)^{\alpha-1} v_{n}(t) d t\right| \\
\quad \leq\left|\frac{1}{\Gamma(\alpha)} \int_{0}^{\tau}\left((s-t)^{\alpha-1}-(\tau-t)^{\alpha-1}\right) v_{n}() d t\right|+\left|\frac{1}{\Gamma(\alpha)} \int_{\tau}^{s}(s-t)^{\alpha-1} v_{n}(t) d t\right| .
\end{array}
$$

Note that, the right side of the inequality towards to zero when $\tau \rightarrow s$. So, the sequence $\left\{\theta v_{n}\right\}$ is equi-continuous and so by using the Arzela-Ascoli theorem there is a uniformly convergent subsequence. Thus, there exists a subsequence of $\left\{v_{n}\right\}$ (we show it again by $\left.\left\{v_{n}\right\}\right)$ such that $\theta v_{n} \rightarrow \theta v$. This implies that $\theta v \in \theta\left(S_{G, y}\right)$. Hence, $\Upsilon y=\theta\left(S_{G, y}\right)$ is compact 
for all $y \in \mathcal{Y}$. Now, we prove that $\Upsilon y$ is convex for each $y \in \mathcal{Y}$. Let $h, h^{\prime} \in \Upsilon y$. Choose $v, v^{\prime} \in S_{G, y}$ such that

$$
\begin{aligned}
h(s)= & \frac{1}{\Gamma(\alpha)} \int_{0}^{s}(s-t)^{\alpha-1} v(t) d t+\frac{-1}{1+\sum_{i=1}^{k} \frac{1}{\Gamma\left(\gamma_{i}+1\right)}} \sum_{i=1}^{k}\left(\frac{1}{\Gamma\left(\alpha-\beta_{i}\right)} \int_{0}^{1}(1-t)^{\alpha-\beta_{i}-1} v(t) d t\right. \\
& \left.+\frac{1}{\Gamma\left(\alpha+\gamma_{i}\right)} \int_{0}^{1}(1-t)^{\alpha+\gamma_{i}-1} v(t) d t\right)
\end{aligned}
$$

and

$$
\begin{aligned}
h^{\prime}(s)= & \frac{1}{\Gamma(\alpha)} \int_{0}^{s}(s-t)^{\alpha-1} v^{\prime}(t) d t+\frac{-1}{1+\sum_{i=1 \frac{1}{\Gamma\left(\gamma_{i}+1\right)}}^{k}} \sum_{i=1}^{k}\left(\frac{1}{\Gamma\left(\alpha-\beta_{i}\right)} \int_{0}^{1}(1-t)^{\alpha-\beta_{i}-1} v^{\prime}(t) d t\right. \\
& \left.+\frac{1}{\Gamma\left(\alpha+\gamma_{i}\right)} \int_{0}^{1}(1-t)^{\alpha+\gamma_{i}-1} v^{\prime}(t) d t\right)
\end{aligned}
$$

for almost all $s \in J$. Let $0 \leq \lambda \leq 1$. Then, we have

$$
\begin{aligned}
\lambda h(s)+(1-\lambda) h^{\prime}(s)= & \frac{1}{\Gamma(\alpha)} \int_{0}^{s}(s-t)^{\alpha-1}\left(\lambda v(t)+(1-\lambda) v^{\prime}(t)\right) d s \\
& +\frac{-1}{1+\sum_{i=1}^{k} \frac{1}{\Gamma\left(\gamma_{i}+1\right)}} \sum_{i=1}^{k}\left(\frac{1}{\Gamma\left(\alpha-\beta_{i}\right)} \int_{0}^{1}(1-t)^{\alpha-\beta_{i}-1}\left(\lambda v(t)+(1-\lambda) v^{\prime}(t)\right) d t\right. \\
& \left.+\frac{1}{\Gamma\left(\alpha+\gamma_{i}\right)} \int_{0}^{1}(1-t)^{\alpha+\gamma_{i}-1}\left(\lambda v(t)+(1-\lambda) v^{\prime}(t)\right) d t\right) .
\end{aligned}
$$

Since $S_{G, y}$ is convex, $\lambda h+(1-\lambda) h^{\prime} \in \Upsilon y$. This completes the proof.

One can check that the fixed point set of $\Upsilon$ is equal to the set of all solutions of the problem (2).

Theorem 11 Suppose that $m \in L^{1}\left(J, \mathbb{R}^{+}\right), G: J \times \mathbb{R}^{2 k+3} \rightarrow \mathcal{P}_{c v, c p}(\mathbb{R})$ is a multifunction such that the map $s \vdash G\left(s, y_{1}, y_{2}, \ldots, y_{2 k+3}\right)$ is measurable,

$$
H\left(G\left(s, y_{1}, y_{2}, \ldots, y_{2 k+3}\right), G\left(s, x_{1}, x_{2}, \ldots, x_{2 k+3}\right)\right) \leq m(s) \sum_{i=1}^{2 k+3}\left|y_{i}-x_{i}\right|
$$

and $\left\|G\left(s, y_{1}, y_{2}, \ldots, y_{2 k+3}\right)\right\|=\sup \left\{|v|: v \in G\left(s, y_{1}, y_{2}, \ldots, y_{2 k+3}\right)\right\} \leq m(s)$ for almost all $s \in J$ and $x_{1}, x_{2}, \ldots, x_{2 k+3}, y_{1}, y_{2}, y_{2 k+3} \in \mathbb{R}$. If Lebesgue measure of the set

$$
\left\{s: \operatorname{dim} G\left(s, y_{1}, y_{2}, \ldots, y_{2 k+3}\right)<1 \text { for some } y_{1}, y_{2}, \ldots, y_{2 k+3} \in \mathbb{R}\right\}
$$

is zero and $l<1$, then the set of all solutions of the problem (2) is infinite dimensional, where lis defined in Theorem 9.

Proof Define the operator $\Upsilon$ by

$$
\Upsilon(y)=\left\{\xi \in \mathcal{Y} \text { : there exists } v \in S_{G, y} \text { such that } \xi(s)=v(s) \text { for all } s \in J\right\} \text {, }
$$


where

$$
\begin{aligned}
v(s)= & \frac{1}{\Gamma(\alpha)} \int_{0}^{s}(s-t)^{\alpha-1} v(t) d t+\frac{-1}{1+\sum_{i=1}^{k} \frac{1}{\Gamma\left(\gamma_{i}+1\right)}} \sum_{i=1}^{k}\left(\frac{1}{\Gamma\left(\alpha-\beta_{i}\right)} \int_{0}^{1}(1-t)^{\alpha-\beta_{i}-1} v(t) d t\right. \\
& \left.+\frac{1}{\Gamma\left(\alpha+\gamma_{i}\right)} \int_{0}^{1}(1-t)^{\alpha+\gamma_{i}-1} v(t) d t\right) .
\end{aligned}
$$

By using Lemma 10, $\Upsilon x \in \mathcal{P}_{c p, c v}(\mathcal{Y})$ for each $y \in \mathcal{Y}$. Like to the Theorem $9, \Upsilon$ is contraction. we show that $\operatorname{dim} \Upsilon y>m$ for each $y \in \mathcal{Y}$ and $m \geq 1$. Let $y \in \mathcal{Y}$ and

$$
F(s)=G\left(s, y(s),(\phi y)(s),(\psi y)(s),{ }^{c} D^{\beta_{1}} y(s),{ }^{c} D^{\beta_{2}} y(s), \ldots,{ }^{c} D^{\beta_{k}} y(s), I^{\gamma_{1}} y(s), I^{\gamma_{2}} y(s), \ldots, I^{\gamma_{k}} y(s)\right)
$$

for all $s \in J$. By using Lemma 4, there exist linearly independent measurable selections $v_{1}, \ldots, v_{m}$ for $F$. Put

$$
\begin{aligned}
h_{i}(s) & =\frac{1}{\Gamma(\alpha)} \int_{0}^{s}(s-t)^{\alpha-1} v_{i}(t) d t+\frac{-1}{1+\sum_{i=1}^{k} \frac{1}{\Gamma\left(\gamma_{i}+1\right)}} \sum_{i=1}^{k}\left(\frac{1}{\Gamma\left(\alpha-\beta_{i}\right)} \int_{0}^{1}(1-t)^{\alpha-\beta_{i}-1} v_{i}(t) d t\right. \\
& \left.+\frac{1}{\Gamma\left(\alpha+\gamma_{i}\right)} \int_{0}^{1}(1-t)^{\alpha+\gamma_{i}-1} v(t)_{i} d t\right)
\end{aligned}
$$

for $i=1, \ldots, m$. Assume that $\sum_{i=1}^{m} a_{i} h_{i}(s)=0$ for almost $s \in J$. By using the Caputo derivatives, we get $\sum_{i=1}^{m} a_{i} v_{i}(s)=0$ for almost $s \in J$. Hence, $a_{1}=\ldots, a_{n}=0$. This implies that $h_{1}, \ldots, h_{m}$ are linearly independent. Thus, $\operatorname{dim} \Upsilon y \geq m$. Now by using Theorem 5 , the set of fixed points of $\Upsilon$ is infinite dimensional.

\section{Conclusions}

The existence of solution for fractional differential inclusions is an important task which can be used successfully in solving real world problems from many fields of science and engineering. Thus, in our paper we analyze firstly the existence of solution of a given class of fractional hybrid differential inclusions. An example was give in order to show the reported results Secondly we concentrate our attention on proving the existence and dimension of the solution set for some fractional differential inclusions. These results are useful for the numerical studies involving the investigated equations.

\section{Authors' contributions}

All authors carried out the proofs of the main results. All authors read and approved the final manuscript.

\section{Author details}

${ }^{1}$ Department of Mathematics, Cankaya University, Ogretmenler Cad. 14, 06530 Balgat, Ankara, Turkey. ${ }^{2}$ Institute of Space Sciences, Magurele, Bucharest, Romania. ${ }^{3}$ Department of Mathematics, Azarbaijan Shahid Madani University, Azarshahr, Tabriz, Iran. ${ }^{4}$ Department of Mathematics, King Saud University, Riyadh, Saudi Arabia.

\section{Acknowlegements}

The research is supported by a grant from the Research Center of the Center for Female Scientific and Medical Colleges, Deanship of Scientific Research, King Saud University. The authors are also thankful to visiting professor program at King Saud University for support.

\section{Competing interests}

The authors declare that they have no competing interests. 
References

Agarwal RP, Ahmad B, Alsaedi A, Shahzad N (2013) Dimension of the solution set for fractional defferential inclusions. J Nonlinear Convex Anal 14:319-329

Ahmad B, Ntouyas SK, Alsedi A (2013) On fractional differential inclusions with anti-periodic type integral boundary conditions. Bound Value Probl 2013:82

Atangana A (2016) On the new fractional derivative and application to nonlinear Fisher's reaction-diffusion equation. Appl Math Comput 273:948-956

Atangana A, Alkahtani BST (2016) New model of groundwater flowing within a confine aquifer: application of CaputoFabrizio derivative. Arab J Geosci 9:8

Atangana A, Koca I (2016a) On the new fractional derivative and application to nonlinear Baggs and Freedman model. J Nonlinear Sci Appl 9(5):2467-2480

Atangana A, Koca I (2016b) Chaos in a simple nonlinear system with Atangana-Baleanu derivatives with fractional order. Chaos Solit Fractals 1-8

Aubin J, Ceuina A (1984) Differential inclusions: set-valued maps and viability theory. Springer, Berlin

Baleanu D, Agarwal RP, Mohammadi H, Rezapour S (2013a) Some existence results for a nonlinear fractional differential equation on partially ordered Banach spaces. Bound Value Probl 2013:112

Baleanu D, Mohammadi H, Rezapour S (2013b) The existence of solutions for a nonlinear mixed problem of singular fractional differential equations. Adv Differ Eq 2013:359

Baleanu D, Mohammadi H, Rezapour S (2013c) Some existence results on nonlinear fractional differential equations. Philos Trans R Soc A 371:20120144

Benchohra M, Hamidi N (2010) Fractional order differential inclusions on the Half-Lin. Surv Math Appl 5:99-111 Berinde V, Pacurar M (2013) The role of the Pompeiu-Hausdorff metric in fixed point theory. Creative Math Inform 22(2):35-42

Bragdi M, Debbouche A, Baleanu D (2013) Existence of solutions for fractional differential inclusions with separated boundary conditions in Banach space. Adv Math Phys (2013) Article ID 426061

Chai G (2013) Existence results for anti-periodic boundary value problems of fractional differential equations. Adv Differ Eq 2013:306

Covitz H, Nadler S (1970) Multivalued contraction mappings in generalized metric spaces. Isr J Math 8:5-11

Deimling K (1992) Multi-valued differential equations. Walter de Gruyter, Berlin

Dhage BC (2006) Multi-valued operators and fixed point theorems in Banach algebras. Taiwan J Math 10(4):1024-1045

Kilbas AA, Srivastava HM, Trujillo JJ (2006) Theory and applications of fractional differential equations. North-Holland Mathematics Studies, Elsevier Science, New York

Kisielewicz M (1991) Differential inclusions and optimal control. Kluwer, Dordrecht

Lasota A, Opial Z (1965) An application of the Kakutani-Ky Fan theorem in the theory of ordinary differential equations. Bull Acad Pol Sci Set Sci Math Astron Phys 13:781-786

Nieto JJ, Ouahab A, Prakash P (2013) Extremal solutions and relaxation problems for fractional differential inclusions. Abstr Appl Anal (2013) Article ID 292643

Ouahab A (2008) Some results for fractional bundary value problem of differential inclusions. Nonlinear Anal 69:3877-3896

Phung PD, Truong LX (2013) On a fractional differential inclusions with integral boundary conditions in Banach space. Fract Calc Appl Anal 16(3):538-558

Podlubny I (1999) Fractional differential equations. Academic Press, New York

Su X (2009) Boundary value problem for a coupled system of nonlinear fractional differential equations. Appl Math Lett 22:64-69

\section{Submit your manuscript to a SpringerOpen ${ }^{\circ}$ journal and benefit from:}

Convenient online submission

- Rigorous peer review

- Immediate publication on acceptance

- Open access: articles freely available online

- High visibility within the field

- Retaining the copyright to your article

Submit your next manuscript at $>$ springeropen.com 